\title{
p53-R273H Sustains ROS, Pro-Inflammatory Cytokine Release and mTOR Activation While Reducing Autophagy, Mitophagy and UCP2 Expression, Effects Prevented by wtp53
}

\author{
Maria Anele Romeo ${ }^{1,2}$, Maria Saveria Gilardini Montani ${ }^{1,2}{ }^{(0}$, Rossella Benedetti ${ }^{1,2}$, Andrea Arena ${ }^{1,2}$, \\ Gabriella D'Orazi ${ }^{3,4}$ (D) and Mara Cirone ${ }^{1,2, *(\mathbb{D}}$ \\ 1 Department of Experimental Medicine, Sapienza University of Rome, 00161 Rome, Italy; \\ mariaanele.romeo@uniroma1.it (M.A.R.); mariasaveria.gilardinimontani@uniroma1.it (M.S.G.M.); \\ benedetti.1589832@studenti.uniroma1.it (R.B.); aarena026@gmail.com (A.A.) \\ 2 Laboratory Affiliated to Istituto Pasteur Italia-Fondazione Cenci Bolognetti, 00161 Rome, Italy \\ 3 Department of Research, IRCCS Regina Elena National Cancer Institute, 00144 Rome, Italy; gdorazi@unich.it \\ 4 Department of Neurosciences, Images and Clinical Sciences, University "G. d'Annunzio", 66013 Chieti, Italy \\ * Correspondence: mara.cirone@uniroma1.it; Tel.: +39-06-4997-3319; Fax: + 39-064-456-229
}

Citation: Romeo, M.A.; Gilardini Montani, M.S.; Benedetti, R.; Arena, A.; D'Orazi, G.; Cirone, M. p53-R273H Sustains ROS, Pro-Inflammatory Cytokine Release and mTOR Activation While Reducing Autophagy, Mitophagy and UCP2 Expression, Effects Prevented by wtp53. Biomolecules 2021, 11, 344. https://doi.org/10.3390/ biom11030344

Academic Editor: Jacques Baudier

Received: 30 December 2020

Accepted: 20 February 2021

Published: 24 February 2021

Publisher's Note: MDPI stays neutral with regard to jurisdictional claims in published maps and institutional affiliations.

Copyright: (c) 2021 by the authors. Licensee MDPI, Basel, Switzerland. This article is an open access article distributed under the terms and conditions of the Creative Commons Attribution (CC BY) license (https:/ / creativecommons.org/licenses/by/ $4.0 /)$.
Abstract: $p 53$ is the most frequently mutated or inactivated gene in cancer, as its activity is not reconcilable with tumor onset and progression. Moreover, mutations in the $p 53$ gene give rise to mutant proteins such as p53-R273H that, besides losing the wild type p53 (wtp53) capacity to safeguard genome integrity, may promote carcinogenesis, mainly due to its crosstalk with prooncogenic pathways. Interestingly, the activation of oncogenic pathways is interconnected with reactive oxygen species (ROS) and the release of pro-inflammatory cytokines that contribute to create an inflammatory/pro-tumorigenic milieu. In this study, based on experiments involving p53- $\mathrm{R} 273 \mathrm{H}$ silencing and transfection, we showed that this mutant p53 (mutp53) promoted cancer cell survival by increasing intracellular ROS level and pro-inflammatory/immune suppressive cytokine release, activating mTOR, reducing autophagy and mitophagy and downregulating uncoupling protein 2 (UCP2). Interestingly, p53-R273H transfection into cancer cells carrying wtp53 induced none of these effects and resulted in p21 upregulation. This suggests that wtp53 may counteract several pro-tumorigenic activities of $\mathrm{p} 53-\mathrm{R} 273 \mathrm{H}$ and this could explain the lower aggressiveness of cancers carrying heterozygous mutp53 in comparison to those harboring homozygous mutp53.

Keywords: mutp53; wtp53; ROS; cytokines; cancer

\section{Introduction}

Several p53 mutant proteins (mutp53), particularly the contact mutant proteins such as p53-R273H, may act as oncogenes, a behavior that mainly depends on their capacity to interact with oncogenic pathways. These pathways, along with other pro-survival functions, may increase mutp53 stabilization, a prerogative for its oncogenic function [1]. Such interplay occurs, for example, between p53-R273H and mammalian target of rapamycin (mTOR) which engages a positive regulatory circuit where mutp53 activates mTOR and this pathway in turn sustains mutp53 expression level by inhibiting autophagy and thus preventing its degradation through this route [2]. Another pathway that may be activated by p53 mutant proteins including p53- $\mathrm{R} 273 \mathrm{H}$ is signal transducer and activator of transcription 3 (STAT3) which may increase mutp53 stability by upregulating heat shock protein 90 (HSP90) and contributing to the activation of the mevalonate pathway [3-5]. The pro-survival effects of p53- $\mathrm{R} 273 \mathrm{H}$ also depend on its capacity to increase intracellular reactive oxygen species (ROS) [6]. Indeed, ROS may sustain cell survival, for example by activating oncogenic pathways such as phosphatidylinositol 3-kinase/ Protein kinase $\mathrm{B} /$ mammalian target of the rapamycin (PI3K/AKT/mTOR) [7] that also promote the release of pro-inflammatory/immune suppressive cytokines including interleukin 6 (IL-6) 
and interleukin 10 (IL-10) [8,9]. Promoting the release of pro-inflammatory cytokines is another oncogenic trait of mutp53 that creates an inflammatory/immune suppressive milieu which strongly promotes tumorigenesis [10]. Interestingly, it is known that most of the effects induced by mutp53 proteins are opposite to those mediated by wild type p53 (wtp53). This also occurs with respect to the activation of mevalonate [11,12], STAT3 [3,13], mTOR [14] and pro-survival pathways, that, as mentioned above, play a crucial role in sustaining mutp53 stability. While wtp53 has been reported to reduce ROS levels, i.e., interfering by with nicotinamide adenine dinucleotide phosphate (NADPH) oxidase (NOX) 4 expression in the course of transforming growth factor beta (TGF- $\beta$ ) treatment [15], mutp53, in the same condition, upregulates NOX4 and increases the level of ROS [16]. Both wtp53 and mutp53 may regulate pro-oxidant and antioxidant responses, although wtp53 seems to activate the former rather than the latter [17]. Nuclear factor erythroid 2-related factor (Nrf2), the most important transcription factor regulating the antioxidant response, has been shown to interact with both wtp53 and mutp53. Nrf2 is a double face molecule, as it is able to prevent carcinogenesis but also promote cancer resistance to anti-cancer therapies $[18,19]$. As a result of these opposite roles in carcinogenesis, Nrf2 can be upregulated by wtp53 for cancer prevention and by mutp 53 for cancer survival, particularly in the course of anti-cancer therapy. Another process involved in ROS regulation that may be contrarily regulated by mutp53 and wtp53 is autophagy, a catabolic route essential for cellular homeostasis. Indeed, mutp53 is known to reduce autophagy, while wtp53 in most of cases induces it, although its role may depend on its intracellular localization [20]. Besides bulk autophagy, mitophagy plays a pivotal role in restraining ROS increases, even if how mutp53 and wtp53 affect this specific process is still under investigation.

In this study, we performed p53- $2273 \mathrm{H}$ silencing and overexpression experiments to investigate the role of this mutp53 on cell survival and correlated it with the level of intracellular ROS, cytokine production, mTOR activation, autophagy, mitophagy and the expression of uncoupling protein 2 (UCP2). p53-R273H overexpression was induced in p53 null, p53 knock out $(\mathrm{K} / \mathrm{O})$ and wtp53 cancer cells. Interestingly, in the latter cells, p53-R273H led to the activation of wtp53/p21 which prevented all pro-tumorigenic effects induced by this mutp53. This could explain why cancers harboring heterozygous mutp53 are less aggressive than those carrying homozygous mutp53.

\section{Material and Methods}

\subsection{Cell Culture and Treatments}

Panc1 (human pancreatic cancer cell line, mutp53), ASPC-1 (human pancreatic cancer cell line, p53null) and U87 (human glioblastoma cell line, wtp53) cell lines were grown in RPMI 1640 medium (Thermo Fisher Scientific, Waltham, MA, USA), while HCT116 (human colon cancer cell line, wtp53) and HCT116 p53-/- (human colon cancer cell line, p53 K/O) were maintained in DMEM 1640 medium (Thermo Fisher Scientific, Waltham, MA, USA), both supplemented with $10 \%$ fetal bovine serum (FBS) (Corning, NY, USA), L-glutamine, streptomycin $(100 \mu \mathrm{g} / \mathrm{mL})$ (Corning, NY, USA), and penicillin $(100 \mathrm{U} / \mathrm{mL})$ (Corning, NY, USA) in $5 \% \mathrm{CO}_{2}$ at $37^{\circ} \mathrm{C}$. Cells were always detached using Trypsin-EDTA solution (Biological Industries, Cromwell, CT, USA).

In some experiments, Panc1 cells were plated in 6-well plates at a density of $2 \times 10^{5}$ cells/well in $2 \mathrm{~mL}$. The following day, cells were treated with N-acetylcysteine (NAC) (5 mM; Sigma Aldrich, St Louis, MO, USA) for $24 \mathrm{~h}$. Untreated cells were used as controls (CT).

\section{2. p53 Silencing, mutp53 and wtp53 Transfection}

Panc1 cells were seeded into 6-well plates at a density of $2 \times 10^{5}$ cells/well and transfected with empty vector (p-Super) or sip53 plasmid (p-Super-p53) [21] for p53 knockdown the following day using Lipofectamine 2000 (Invitrogen, Waltham, MA, USA) according to the manufacturer's instructions. After $48 \mathrm{~h}$, a Trypan blue assay was performed, and cells were recovered for further analysis. 
ASPC-1, U87, HCT116 wt and HCT116 p53-/- cells were seeded into 6-well plates at a density of $2 \times 10^{5}$ cells/well and were transfected with pcDNA3- p53R273H vector (kindly provided by Prof. D'Orazi) or with empty vector (EV) the following day using Lipofectamine 2000 (Invitrogen) according to the manufacturer's instructions. In some experiments, HCT116 p53-/- cells were co-transfected with pcDNA3- p53R273H vector and pcDNA3 p53 wt vector (kindly provided by Prof. D'Orazi) or with empty vector (EV) using Lipofectamine 2000 (Invitrogen). After $24 \mathrm{~h}$, a Trypan blue assay was performed and cells were recovered for further analysis. In some other experiments, HCT116 wtp53 cells were pre-treated with pifithrin- $\alpha(\mathrm{P})(30 \mu \mathrm{M}$, Sigma) or UC2288-p21 inhibitor (p21 inh) ( $5 \mu \mathrm{M}$, Millipore, Burlington, MA, USA). After $24 \mathrm{~h}$, a Trypan blue assay was performed and cells were recovered for further analysis.

\subsection{Cell Viability}

Cell viability was evaluated using a Trypan blue (Sigma Aldrich) exclusion assay. Cells were counted by light microscopy using a Neubauer hemocytometer. The experiments were performed in triplicate and repeated at least three times.

\subsection{Measurement of Intracellular Reactive Oxygen Species (ROS) Production}

To measure reactive oxygen species (ROS) production, $10 \mu \mathrm{M}$ 2,7-dichlorofluorescein diacetate (DCFDA; Sigma-Aldrich D6883) was added to cell cultures for $15 \mathrm{~min}$ and live cells, gated according to their forward scatter (FSC) and side scatter (SSC) properties, were analyzed on a FACScalibur flow cytometer (BD Transduction Laboratories, Franklin Lakes, NJ, USA ) using CELLQuest Pro software (version 6.0, BD Biosciences, Franklin Lakes, NJ, USA). For each analysis, 10,000 events were recorded.

\subsection{Western Blot Analysis}

Following transfections and treatments, cells were washed in $1 \times$ PBS, lysed in RadioImmunoprecipitation Assay (RIPA)buffer (150 mM NaCl, 1\% NP-40, $50 \mathrm{mM}$ Tris- $\mathrm{HCl}$ (pH 8), $0.5 \%$ deoxycholic acid, $0.1 \%$ SDS, protease and phosphatase inhibitors) and centrifuged at $14,000 \mathrm{rpm}$ for $20 \mathrm{~min}$ at $4{ }^{\circ} \mathrm{C}$. The protein concentration was measured using the Bio-Rad Protein Assay (BIO-RAD laboratories $\mathrm{GmbH}$, Hercules, CA, USA) and $12 \mu \mathrm{g}$ of protein were subjected to electrophoresis on 4-12\% NuPage Bis-Tris gels (Life Technologies, Carlsbad, CA, USA) according to the manufacturer's instructions. Then, the gels were transferred onto nitrocellulose membranes (BIO-RAD, Hercules, CA, USA) for $2 \mathrm{~h}$ in Tris-Glycine buffer. The membranes were blocked in $1 \times$ PBS $+0.1 \%$ Tween 20 solution containing $3 \%$ bovine serum albumin BSA (Serva, Reno, NV, USA), probed with specific antibodies (see Section 2.6 below) and developed using Electrochemiluminescence ECL Blotting Substrate (Advansta, San Josè, CA, USA).

\subsection{Antibodies}

To evaluate the expression of proteins in Western blot analysis, we used the following antibodies: mouse monoclonal anti-p53 (1:100) (clone DO-1, Santa Cruz Biotechnology Inc., sc-126, Heidelberg, Germany), rabbit monoclonal anti-phospho-4E-BP1 (Thr37/46) (236B4) (1:1000) (Cell Signaling, 2855, Danvers, MA, USA), rabbit monoclonal anti-4EBP1 (1:2000) (Proteintech, 60246-1-Ig, Rosemont, IL, USA), mouse monoclonal anti-p62 (SQSMT1) (1:1000) (BD Transduction Laboratories, 610832, Franklin Lakes, NJ, USA), mouse monoclonal anti-p21 (1:100) (clone F-8, Santa Cruz Biotechnology Inc., sc-271610), mouse monoclonal anti-cyclin D1 (1:100) (clone A-12, Santa Cruz Biotechnology Inc., sc8396), mouse monoclonal anti-HADHA (1:100) (clone F-8, Santa Cruz Biotechnology Inc., sc-374497), mouse monoclonal anti-UCP2 (1:100) (clone G-6, Santa Cruz Biotechnology Inc., sc-390189), mouse monoclonal anti-Nrf2 (1:100) (clone A-10, Santa Cruz Biotechnology Inc., sc-365949), and mouse monoclonal anti-NQO1 (clone H-9, Santa Cruz Biotechnology Inc., sc-376023). Mouse monoclonal anti- $\beta$-actin (1:10000) (Sigma Aldrich) was used as a loading control. Goat anti-mouse IgG-HRP (1:30000) (Bethyl Laboratories, A90-116P) 
ad goat anti-rabbit IgG-HRP (1:30000) (Bethyl Laboratories, A120-101P, Montgomery, TX, USA) were used as secondary antibodies. All primary and secondary antibodies were diluted in PBS $+0.1 \%$ Tween20 solution containing 3\% BSA (SERVA, Reno, NV, USA).

\subsection{Chemiluminescent Immunometric Assay}

After culturing for $24 \mathrm{~h}$, supernatants derived from transfections were analyzed using a magnetic Luminex assay using a human pre-mixed multi-analyte kit (R\&D Systems Bio-Techne, Minneapolis, MN, USA) according to the manufacturer's instructions.

\subsection{Indirect Immunofluorescence Assay (IFA)}

An indirect immunofluorescence assay was used to analyze HADHA in p53wt and p53-/- HCT116 cells $24 \mathrm{~h}$ transfected with mutp53-R273H. Cells were applied onto multispot microscope slides and air-dried, then fixed with $2 \%$ paraformaldehyde (Electron Microscopy Science, 157-8, Hatfield, PA, USA) for $30 \mathrm{~min}$ and permeabilized with $0.1 \%$ Triton X-100 (Sigma Aldrich, T-8787) for $5 \mathrm{~min}$. Cells were then incubated with a primary monoclonal antibody against HADHA (clone F-8, Santa Cruz Biotechnology Inc., sc-374497) for $1 \mathrm{~h}$ at room temperature, washed with PBS and incubated with a polyclonal conjugated-Cy3 sheep anti-mouse antibody (Jackson ImmunoResearch, 515-165-062, Ely, UK) for $30 \mathrm{~min}$. Cells were also stained with $1 \mu \mathrm{g} / \mathrm{mL} \mathrm{4} 4^{\prime}, 6^{\prime}$-diamidino-2-phenylindole (DAPI) (Sigma-Aldrich), mounted with glycerol:PBS (1:1) and observed on a fluorescence microscope (Olympus BX53, Norfolk, VA, USA).

\subsection{Statistical Analysis}

Results are represented as the mean \pm standard deviation (SD) of at least three independent experiments and a two-tailed Student's t-test was used to demonstrate statistical significance. Differences were considered statistically significant when $p$-values were at least $<0.05$.

\section{Results}

3.1. p53-R273H Silencing Reduces Cell Survival, Intracellular ROS, Cytokine Release and mTOR Activation While Inducing Autophagy in Panc1 Cells

We evaluated the role of mutp53 on cell survival by silencing it using a specific siRNA in Panc1 cells, a pancreatic cell line harboring the $p 53-R 273 H$ mutation. We found that p53-R273H silencing reduced cell survival in comparison to the scramble treatment (Figure $1 \mathrm{~A}, \mathrm{~B})$. We also found that this effect was correlated with a reduction in intracellular ROS (Figure 1C,D), whose role in cell survival has been previously reported [6]. Here, we confirmed these findings by using n-acetylcysteine (NAC), a ROS scavenger, that indeed impaired Panc1 cell survival similarly to mutp53 silencing (Figure 1E). As mTOR is one of the oncogenic pathways activated by mutp53 [2], and given that ROS contribute to the activation of this oncogenic pathway [7], we then evaluated the phosphorylation status of 4E-BP1, an mTOR target in Panc1 cells undergoing mutp53 silencing or NAC treatment. As shown in Figure 1F,G, both treatments reduced the activation of 4E-BP1, suggesting that ROS could play a role in mTOR activation induced by p53-R273H. mTOR is the master regulator of autophagy and its activation by mutp53 has been reported to prevent its degradation through autophagy [22]. Therefore, we also evaluated autophagic flux in scramble and mutp53 silenced Panc1 cells by assessing the expression level of p62/sequestosome 1 (SQSTM1), a molecule that is mainly degraded through autophagy. As shown in Figure 1H, the expression level of p62/SQSTM1 was reduced in mutp53silenced Panc1 cells, suggesting that its knockdown removed the block on autophagy. Altogether, these results suggest that p53-R273H silencing reduces cells survival, ROS and mTOR activation while restoring autophagy in Panc1 cells. 
A
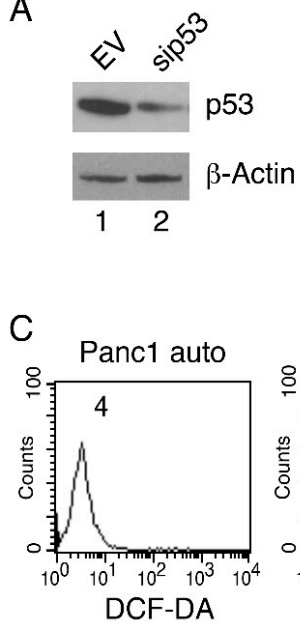

E

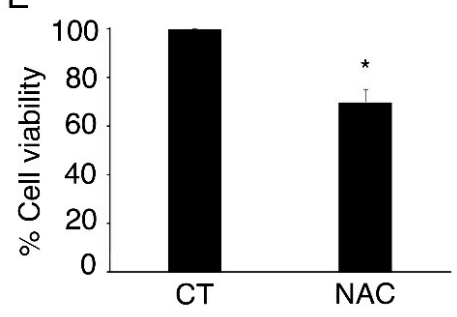

B

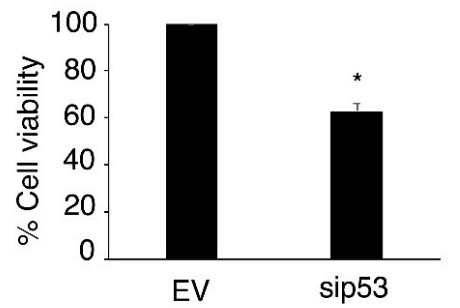

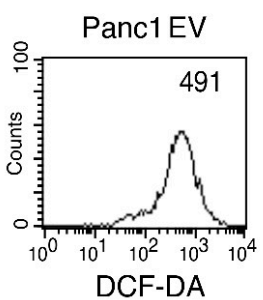
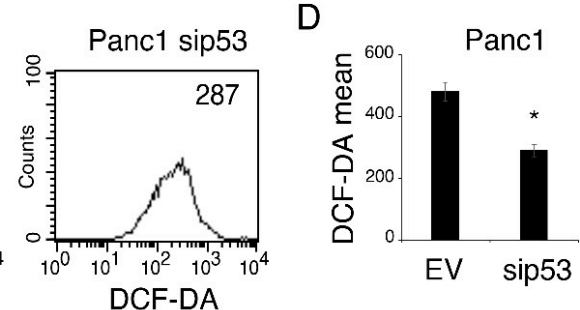

$\mathrm{F}$
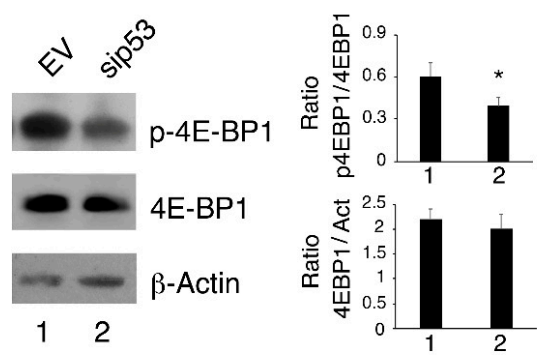

$\mathrm{G}$
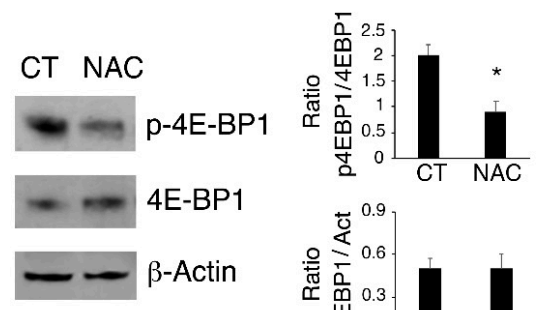

$\mathrm{H}$
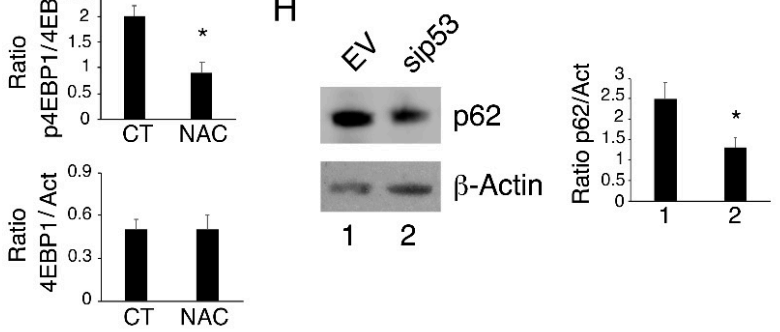

12

Figure 1. p53-R273H silencing reduces cell survival and intracellular reactive oxygen species (ROS) and induces autophagy in Panc1 cells. Panc1 cells were transfected with empty vector (EV, 1) or the sip53 vector $($ sip53, 2) and after $48 \mathrm{~h}(\mathrm{~A})$ p53 expression was evaluated by Western blot analysis. Actin was used as loading control; the histograms represent the mean + standard deviation (SD) of the densitometric analysis of the ratio of p53 to actin. (B) Cell survival was evaluated by Trypan blue exclusion assay. The histograms represent the percentage of cell viability relative to the control; data are represented as the mean $+\mathrm{SD}$ of more than 3 experiments. ${ }^{*} p$ value $<0.05$. (C) Intracellular ROS level was measured by FACS analysis using DCF-DA. Mean fluorescence intensity (MFI) is indicated. One representative experiment out of three is reported. Auto $=$ autofluorescence. $\left(\right.$ D) Histograms represent the mean of the MFI of DCF-DA + SD. ${ }^{*} p$ value $<0.05$. (E) The survival of Panc1 cells treated with n-acetylcysteine (NAC) (5mM) for $24 \mathrm{~h}$ was evaluated by Trypan blue exclusion. The histograms represent the percentage of cell viability relative to the control; data are represented as the mean $+\mathrm{SD}$ of more than 3 experiments. ${ }^{*} p$ value $<0.05$. (F) The expression of p-4E-BP1 and 4-E-BP1 were analyzed by Western blot analysis. Actin was used as the loading control. The histograms represent the mean $+\mathrm{SD}$ of the densitometric analysis of the ratio between the protein and the appropriate control. ${ }^{*} p$ value $<0.05$. (G) Protein expression of p-4E-BP1 and 4-E-BP1 in NAC-treated or untreated cells were analyzed by Western blot. Actin was used as the loading control. The histograms represent the mean $+\mathrm{SD}$ of the densitometric analysis of the ratio between the protein and the appropriate control. * $p$ value $<0.05$. (H) The expression of $\mathrm{p} 62$ in Panc1 cells transfected with empty vector $(\mathrm{EV}, 1)$ or the sip53 vector $($ sip53, 2) was analyzed by Western blot. Actin was used as the loading control. The histograms represent the mean + SD of the densitometric analysis of the ratio between the protein and the appropriate control. ${ }^{*} p$ value $<0.05$. 


\section{2. mutp53-R273H Overexpression Increases Cell Survival, ROS and Inflammatory Cytokine Release in p53 Null Cells; Effects Were Not Observed in wtp53 Cells}

We then investigated if the transfection of p53- $2273 \mathrm{H}$ into cancer cells that do not carry this mutation could induce opposite effects compared to $\mathrm{p} 53-\mathrm{R} 273 \mathrm{H}$ silencing. We found that $\mathrm{p} 53-\mathrm{R} 273 \mathrm{H}$ overexpression (Figure 2A) promoted cell survival (Figure 2B) and increased intracellular ROS (Figure 2C,D) in pancreatic cancer ASPC1 p53-null cells. Interestingly, the overexpression of mutp53 in U87 (Figure 2E), a glioblastoma cell line carrying wtp53, slightly affected cell survival (Figure 2F) and did not increase intracellular ROS (Figure 2G,H). As ROS are strongly inter-connected with the release of pro-inflammatory cytokines and other molecules involved in pro-tumorigenic effects induced by mutp53 [10], we then evaluated inflammatory cytokine release by ASPC1 and U87 cells transfected with p53-R273H or with empty vector (EV). Interestingly, multi-analyte Luminex assay analysis indicated that mutp53 increased the release of pro-inflammatory/immune suppressive cytokines by ASPC1 cells and, once again, this effect was not observed in U87 cells (Figure 2I). Finally, we found that p53-R273H overexpression upregulated the expression level of p21 in U87 cells, while it only slightly affected it in ASPC1 cells (Figure 2L), suggesting that a reactivation of wtp53 by p53-R273H transfection could counteract the pro-survival effects induced by mutp53.

\section{3. p53-R273H Overexpression Increases Cell Survival, ROS and Inflammatory Cytokine Release in HCT116 p53-/- But Not in HCT116 wtp53 Cells}

To investigate the effect of p53-R273H transfection in another cell type and to exclude that the different outcomes observed in p53-null and wtp53-carrying cells (ASPC and U87, respectively) could be due to the different cellular background, we repeated the p53-R273H transfection experiments in HCT116 cells carrying wtp53 and in the same cells with p53 knocked out. We found that p53-R273H transfection (Figure 3A) increased cell survival (Figure 3B) and ROS levels (Figure 3C,D) in HCT116 p53-/- cells and that it did not induce such an effect in HCT116 wtp53 cells (Figure 3E-H) in which, similarly to what was observed in U87 cells, p21 was upregulated (Figure 3I). The role of wtp53 activation in p21 upregulation was then demonstrated by treating HCT116 wtp53 cells with pifithrin- $\alpha$, a p53 inhibitor, which prevented p21 upregulation following $p 53-R 273 H$ transfection (Figure 3I). Next, to further demonstrate the capacity of wtp53 to counteract the effects induced by mutp53, we co-transfected wtp53 and mutp53 into HCT116 p53-/- cells (Figure 3L) and found that p21 was upregulated but cell survival and ROS did not increase (Figure 3M-O). To investigate the mechanism through which the activation of the p53/p21 axis could counteract the pro-survival effect induced by mutp53, we evaluated the expression of cyclin D1 which can be activated by mutp53 and repressed by wtp53 [23,24]. As shown in Figure 4A,B, cyclin D1 expression increased in HCT116 p53-/- cells while it slightly decreased in wtp53 cells; thus, this may be one of the reasons why only the p53-/- cells showed an increase in cell proliferation following mutp53 transfection. Finally, we found that in HCT116 p53-/-cells, p53-R273H promoted the release of pro-inflammatory/immune suppressive cytokines, and again, such an effect was not observed in HCT116 wtp53 cells (Figure 4C,D). Altogether, these results suggest that the activation of wtp53 also prevents several pro-survival effects induced by $\mathrm{p} 53-\mathrm{R} 273 \mathrm{H}$ in cells with a similar background.

\section{4. p53-R273H Overexpression in HCT116 p53-/- Cells Induces mTOR Activation, Reduces} Autophagy and Mitophagy and Downregulates UCP2 Expression

As ROS contribute to the activation of oncogenic pathways such as mTOR [7], the phosphorylation of 4-E-BP1, an mTOR target, was then investigated in HCT116 p53-/and wtp53 cells following p53-R273H transfection. We found that p-4E-BP1 increased in both HCT116 p53-/- cells and HCT116 wtp53 cells (Figure 5A,B). As mTOR is a negative regulator of autophagy, we also found that p62/SQSTM1 accumulated in HCT116 p53-/cells, suggesting a reduction in autophagic flux (Figure 5C,D). 
A
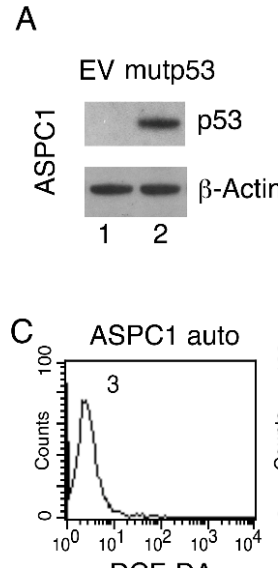

DCF-DA

E

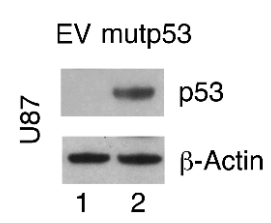

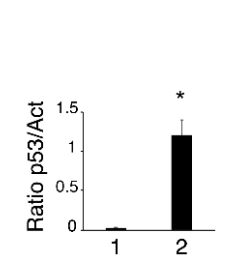
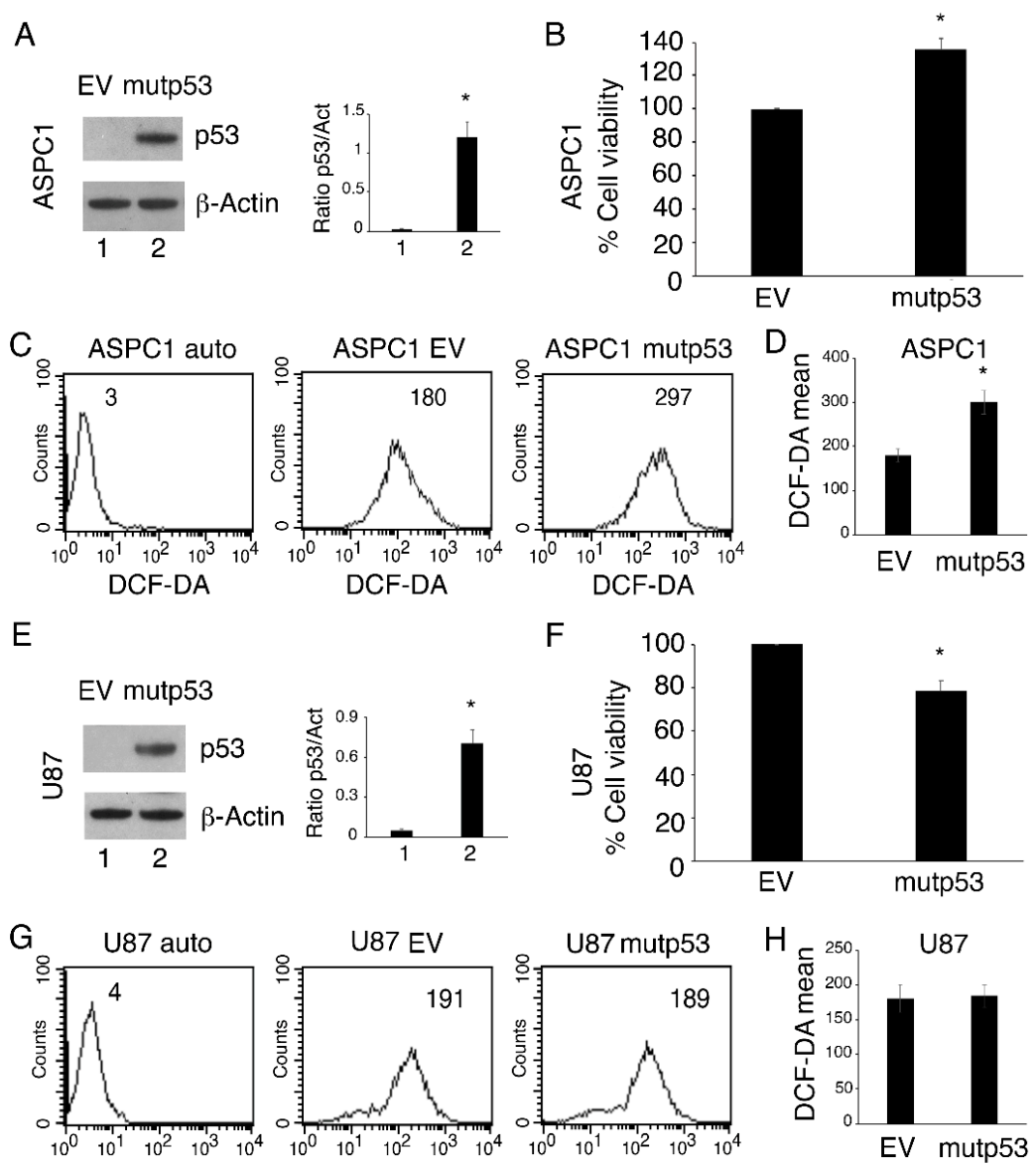

DCF-DA
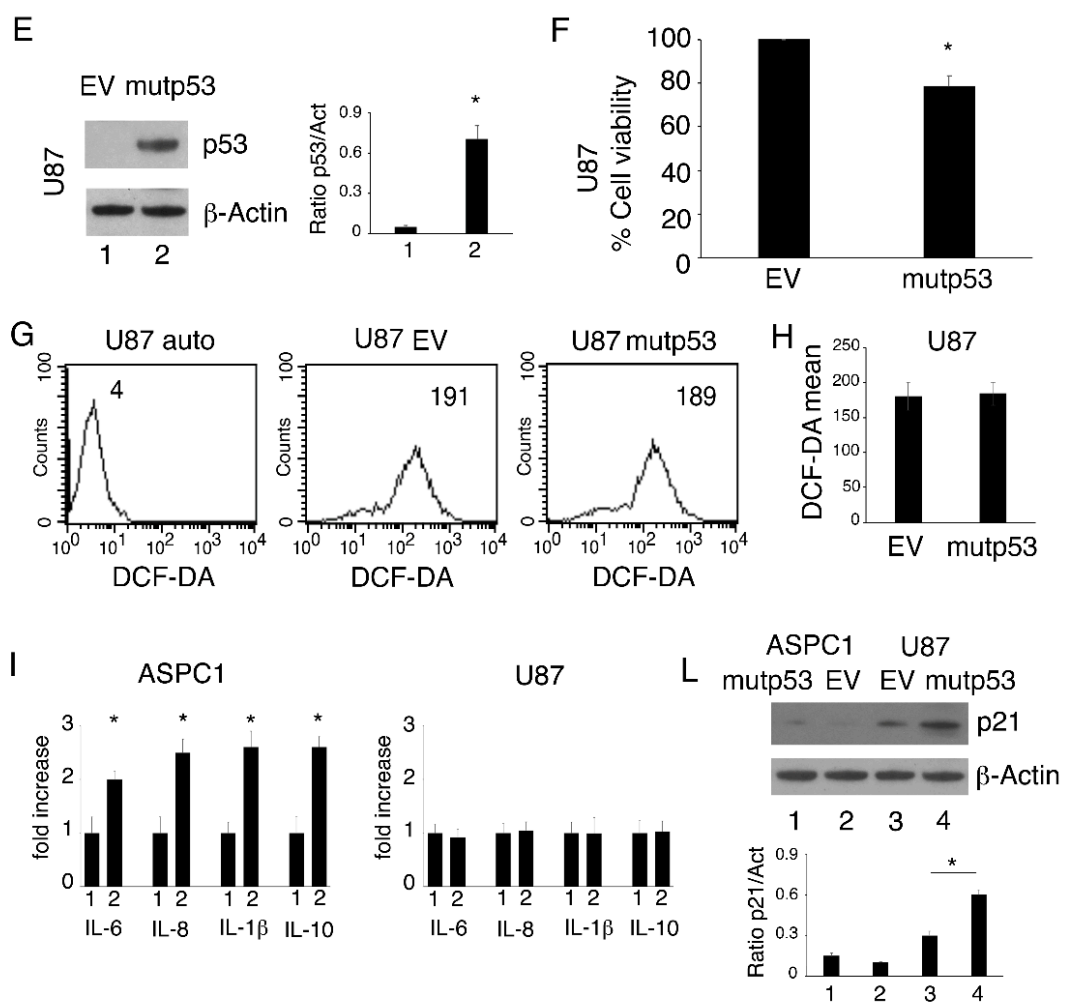

Figure 2. p53-R273H overexpression increases cell survival, ROS, and inflammatory cytokine release in ASPC1 p53-null cells and U87 wtp53-carrying cells. ASPC1 and U87 cell lines were transfected with empty vector (EV,1) or the pcDNA3p53R273H vector (mutp53, 2). After $24 \mathrm{h:} \mathrm{(A)} \mathrm{and} \mathrm{(E)} \mathrm{transfection} \mathrm{was} \mathrm{evaluated} \mathrm{by} \mathrm{Western} \mathrm{blot} \mathrm{analysis} \mathrm{of} \mathrm{the} \mathrm{expression}$ of p53. The histograms represent the mean $+\mathrm{SD}$ of the densitometric analysis of the ratio of p53 to actin. ${ }^{*} p$ value $<0.05$. (B) and (F) Cell survival was evaluated by Trypan blue exclusion assay. The histograms represent the percentage of cell viability relative to the appropriate control; data are represented as the mean $+\mathrm{SD}$ of more than 3 experiments. ${ }^{*} p$ value $<0.05$. (C) and (G) Intracellular ROS level was measured by FACS analysis using DCF-DA to stain cells. Mean fluorescence intensity (MFI) is indicated. One representative experiment out of three is reported. (D) and (H) Histograms represent the mean of the MFI of DCF-DA plus SD. ${ }^{*} p$ value $<0.05$. (I) After $24 \mathrm{~h}$ of culture, supernatants derived from cells transfected with EV or mutp53 were collected and IL-6, IL-8, IL-1 $\beta$ and IL-10 were measured by Luminex assay. Histograms represent the fold increase of the values for each cytokine compared to EV transfection. The results were considered significant $\left(^{*}\right)$ when the $p$ value was $<0.05$. (L) p21 expression was evaluated by Western blot analysis. Actin was used as the loading control. The histograms represent the mean $+\mathrm{SD}$ of the densitometric analysis of the ratio of p21 to actin. * $p$ value $<0.05$. 
A
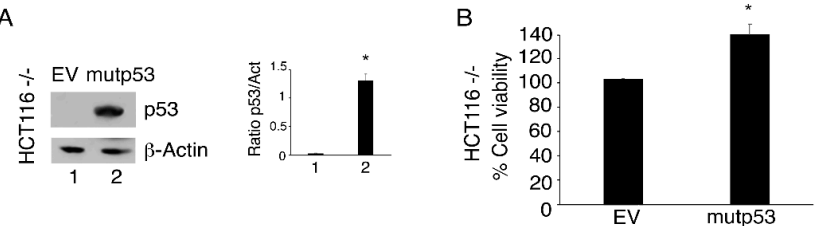

C
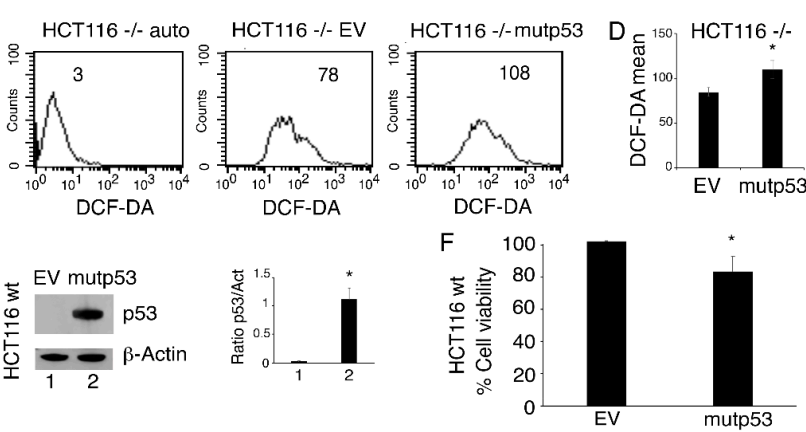

E
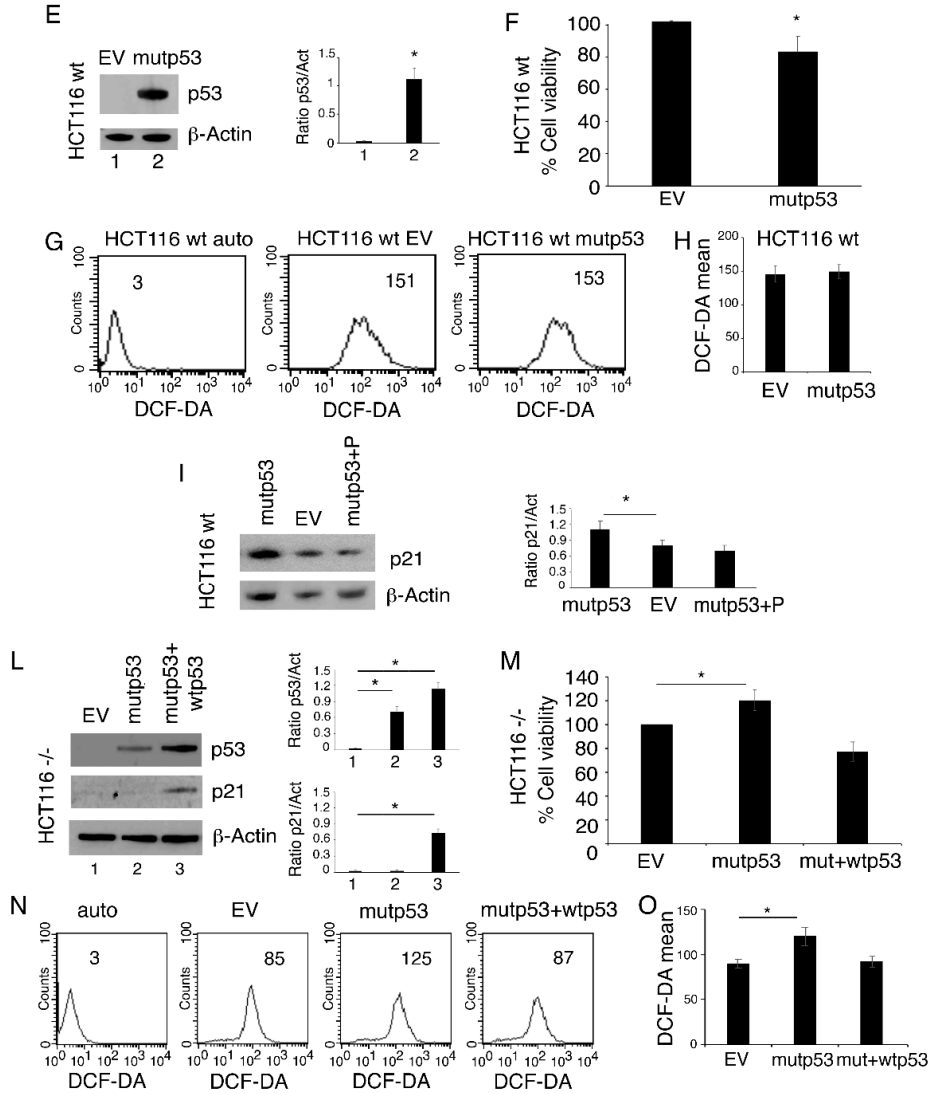

Figure 3. $p 53-R 273 H$ overexpression increases cell survival and ROS in HCT116 p53-/- but not in HCT116 wtp53 cells. HCT116 p53-/- and HCT116 wtp53 cells were transfected with empty vector (EV, 1) or pcDNA3- p53R273H vector (mutp53, 2). In some experiments, HCT116 p53-/- cells were co-transfected with the pcDNA3- p53R273H vector and the pcDNA3 p53wt vector (mutp53 + wtp53, 3). (A) and (E) After $24 \mathrm{~h}$, transfection was evaluated by Western blot analysis to examine the expression of $\mathrm{p} 53$. The histograms represent the mean $+\mathrm{SD}$ of the densitometric analysis of the ratio of $\mathrm{p} 53$ to actin. * $p$ value $<0.05$. (B), (F) and (M) Cell survival was evaluated by Trypan blue exclusion assay. The histograms represent the percentage of cell viability relative to the appropriate control; data are represented as the mean $+\mathrm{SD}$ of more than 3 experiments. The results were considered significant $\left(^{*}\right)$ when $p$ value $<0.05$. (C), (G) and (N) Intracellular ROS level was measured by FACS analysis using DCF-DA to stain cells. Mean fluorescence intensity (MFI) is indicated. One representative experiment out of three is reported. (D), (H) and (O) Histograms represent the mean of the MFI of DCF-DA + SD. ${ }^{*} p$ value $<0.05$. (I) HCT116 wtp53 cells were transfected with empty vector (EV) or pre-treated with pifihtrin- $\alpha(\mathrm{P})(30 \mu \mathrm{M})$ and then transfected with pcDNA3- p53R273H (mutp53) to evaluate p21 expression by Western blot analysis. Controls were untreated. The histograms represent the mean $+\mathrm{SD}$ of the densitometric analysis of the ratio of $\mathrm{p} 21$ to actin. ${ }^{*} p$ value $<0.05$. (L) The expression of p53 and p21 was evaluated by Western blot analysis of co-transfected HCT116 p53-/- (mutp53 + wtp53). The histograms represent the mean $+\mathrm{SD}$ of the densitometric analysis of the ratio of p53 or p21 to actin. ${ }^{*} p$ value $<0.05$. 
A

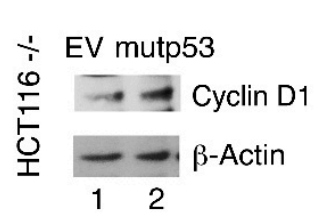

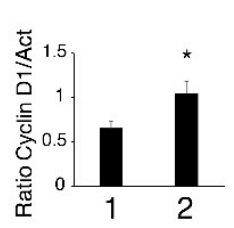

C

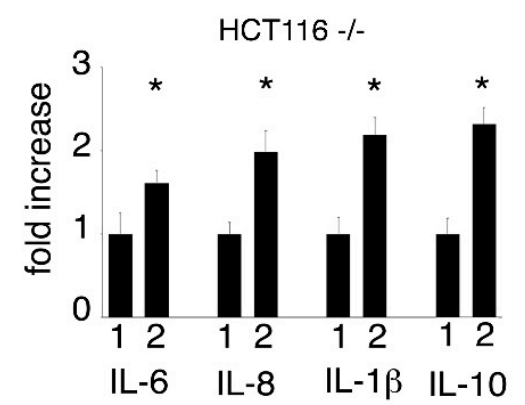

B
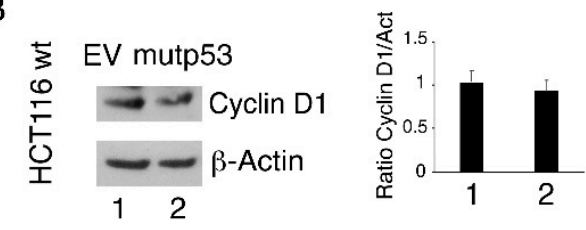

D

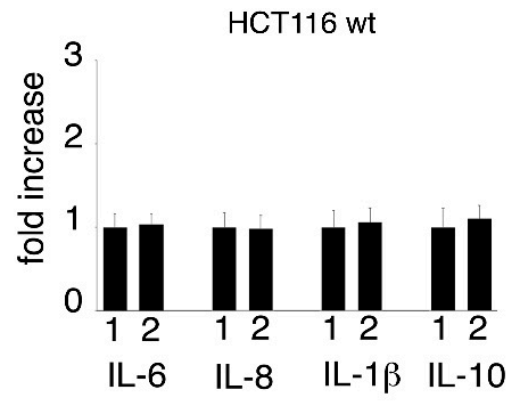

Figure 4. $p 53-R 273 H$ overexpression differently modulates cyclin D1 expression in HCT116 p53-/- and HCT116 wtp53 cells and increases inflammatory cytokine release in HCT116 p53-/- but not in HCT116 wtp53 cells. HCT116 p53-/- and HCT116 wtp53 cells were transfected with empty vector (EV, 1) or the pcDNA3- p53R273H vector (mutp53, 2). (A) and (B) After a $24 \mathrm{~h}$ transfection, the expression of cyclin D1 was evaluated by Western blot analysis. The histograms represent the mean $+\mathrm{SD}$ of the densitometric analysis of the ratio of cyclin D1 to Actin. ${ }^{*} p$ value $<0.05$. (C) and (D) After $24 \mathrm{~h}$ of culture, supernatants derived from HCT116 p53-/- and HCT116 wtp53 cells transfected with EV or mutp53 were collected, and IL-6, IL-8, IL-1 $\beta$ and IL-10 were measured by Luminex assay. Histograms represent the fold increase of the values of each cytokine compared to EV transfected cells. The results were considered significant $\left.{ }^{*}\right)$ when the $p$-value was $<0.05$.

Besides bulk autophagy, a selective form of autophagy known as mitophagy plays a major role in the elimination of damaged mitochondria, which are the main source of ROS. As ROS increased following p53-R273H transfection into cancer cells lacking wtp53, we then evaluated mitophagy in HCT116 p53-/- or mutp53-transfected wtp53 cells. The results shown in Figure 5E-G indicate that the expression level of HADHA, a molecule degraded through mitophagy, increased following p53-R273H transfection in HCT116 p53-/- cells and was slightly affected in HCT116 wtp53 cells as evaluated by Western blot and IFA, respectively. This suggests that like autophagy, mitophagy was also impaired by p53- $273 \mathrm{H}$ transfection into cells lacking wtp53 and could further contribute to the observed ROS increase. To investigate the role of autophagy/mitophagy inhibition in ROS increase, we used chloroquine, an inhibitor of the final steps in the autophagy pathway, in HCT116 wtp53 cells and found that chloroquine treatment also increased ROS in these cells (Figure 5H). Interestingly, mitophagy is also under the control of mTOR, as we recently observed in Kaposi's Sarcoma-associated Herpesvirus (KSHV)-infected Human umbilical vein endothelial cells (HUVECs) cells [25]. One of the proteins playing a key role in ROS production and known to be upregulated by mutp53 is UCP2 [26]; therefore, we next evaluated the impact of p53-R273H transfection on this protein. As shown in Figure 5I,L, UCP2 expression decreased in HCT116 p53-/- cells while it was slightly affected in HCT116 wtp53 cells. Interestingly, UCP2 expression has been correlated with AMP-activated protein kinase (AMPK) [26] and mTOR activation [27]; accordingly, in this study, we observed that UCP2 was downregulated in HCT116 p53-/-cells in which mTOR activity increased. Altogether, these results indicate that autophagy/mitophagy reduction and UCP2 downregulation could be responsible for the observed ROS increases in HCT116 p53-/- cells transfected with mutp53. 
A

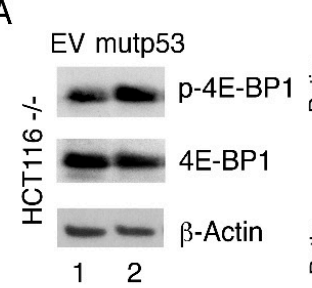

C

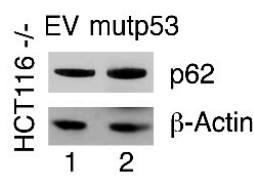

E

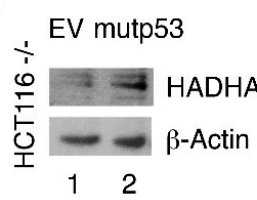

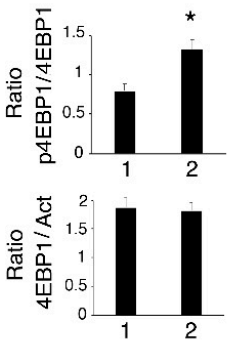
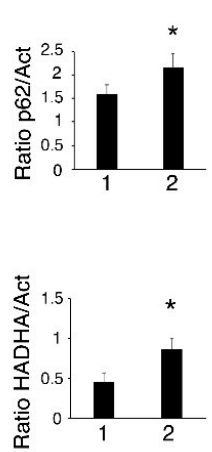

G
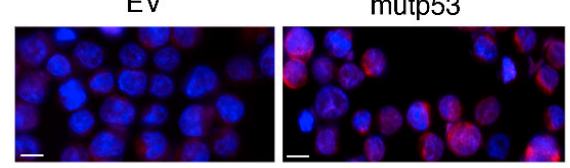

HCT116 -/-

$\mathrm{H}$

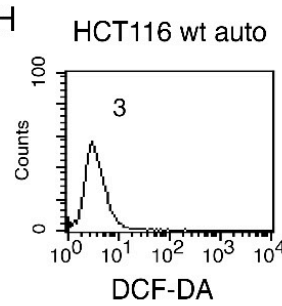

I
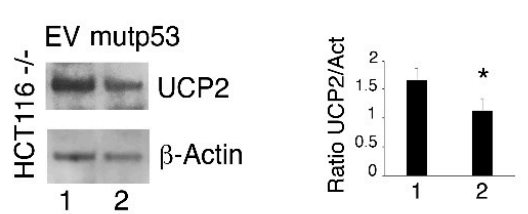
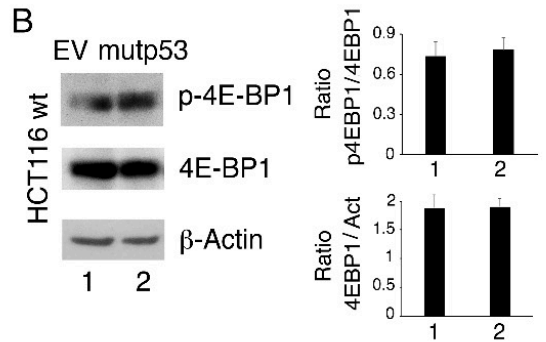

D
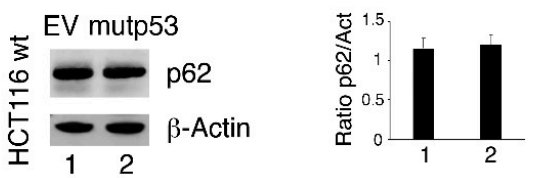

F
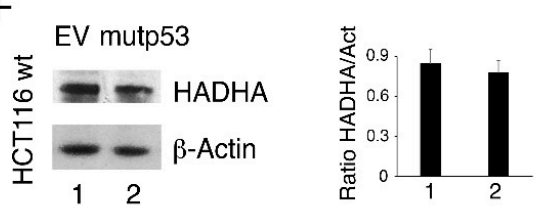

EV
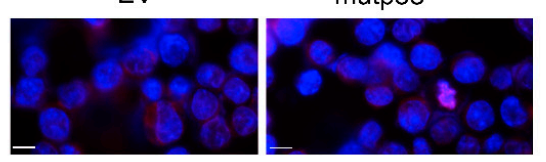

HCT116 wt
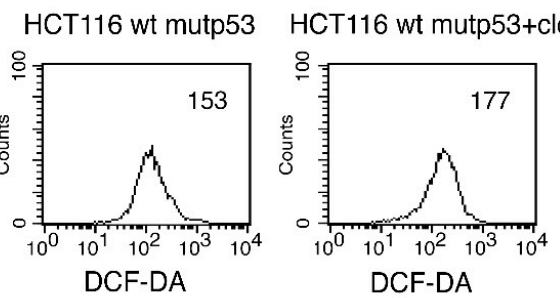

L

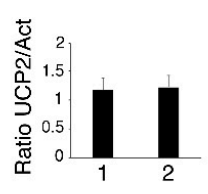

Figure 5. $p 53-R 273 H$ transfection in HCT116 p53-/- cells induces mammalian target of rapamycin (mTOR) activation, reduces autophagy and mitophagy, and downregulates mitochondrial uncoupling proteins 2 (UCP2) expression. HCT116 wtp53 and HCT116 p53-/- cells were transfected with empty vector $(\mathrm{EV}, 1)$ or the pcDNA3- p53R273H vector (mutp53, 2). (A) and (B) After $24 \mathrm{~h}$, the expression of p-4E-BP1 and 4E-BP1 was analyzed by Western blot. Actin was used as the loading control. The histograms represent the mean $+\mathrm{SD}$ of the densitometric analysis of the ratio between the protein and the appropriate control. ${ }^{*} p$ value $<0.05$. (C) and (D) p62 expression and (E) and (F) HADHA expression as evaluated by Western blot analysis. The histograms represent the mean $+\mathrm{SD}$ of the densitometric analysis of the ratio between the protein and the appropriate control. * $p$ value $<0.05$. (G) HADHA expression in mutp53-R273H-transfected HCT116 p53-/and HCT116 wtp53 cells as evaluated by immunofluorescence assay (IFA) after $24 \mathrm{~h}$. Nuclei are stained in blue with DAPI and HADHA in red. Scale bar: $10 \mu \mathrm{m}$. (H) Intracellular ROS level was measured by FACS analysis using DCF-DA to stain cells. Mean fluorescence intensity (MFI) is indicated. One representative experiment out of three is reported. (I) and (L) UCP2 expression was evaluated by Western blot analysis. Actin was used as the loading control. The histograms represent the mean $+\mathrm{SD}$ of the densitometric analysis of the ratio between the protein and the appropriate control. ${ }^{*} p$ value $<0.05$. 
3.5. The Nrf2 Antioxidant Program is Activated in HCT116 p53-/- But Not in HCT116 wtp53 Following p53-R273H Transfection

ROS level is strongly regulated by the antioxidant response, whose main activator is Nrf2. Therefore, we also evaluated the expression of this transcription factor in HCT116 p53-/- cells and HCT116 wtp53 cells following p53-R273H transfection. As shown in Figure 6A,B, Nrf2 was upregulated by p53-R273H transfection in both cell lines, independently of the presence of wtp53. As mutp53 has been reported to modulate the transcriptional program of Nrf2 [28], we then evaluated the expression of $\mathrm{NAD}(\mathrm{P}) \mathrm{H}$ dehydrogenase quinone 1 (NQO1), an Nrf2 target molecule whose expression has been shown to be reduced by mutp53 [29]. We found that the expression of NQO1 was slightly affected in HCT116 p53-/- cells transfected with p53-R273H, while it more clearly increased in HCT116 wtp53 cells (Figure 6A). According to previous findings, NQO1 may engage in a positive feedback loop with wtp53 [18].

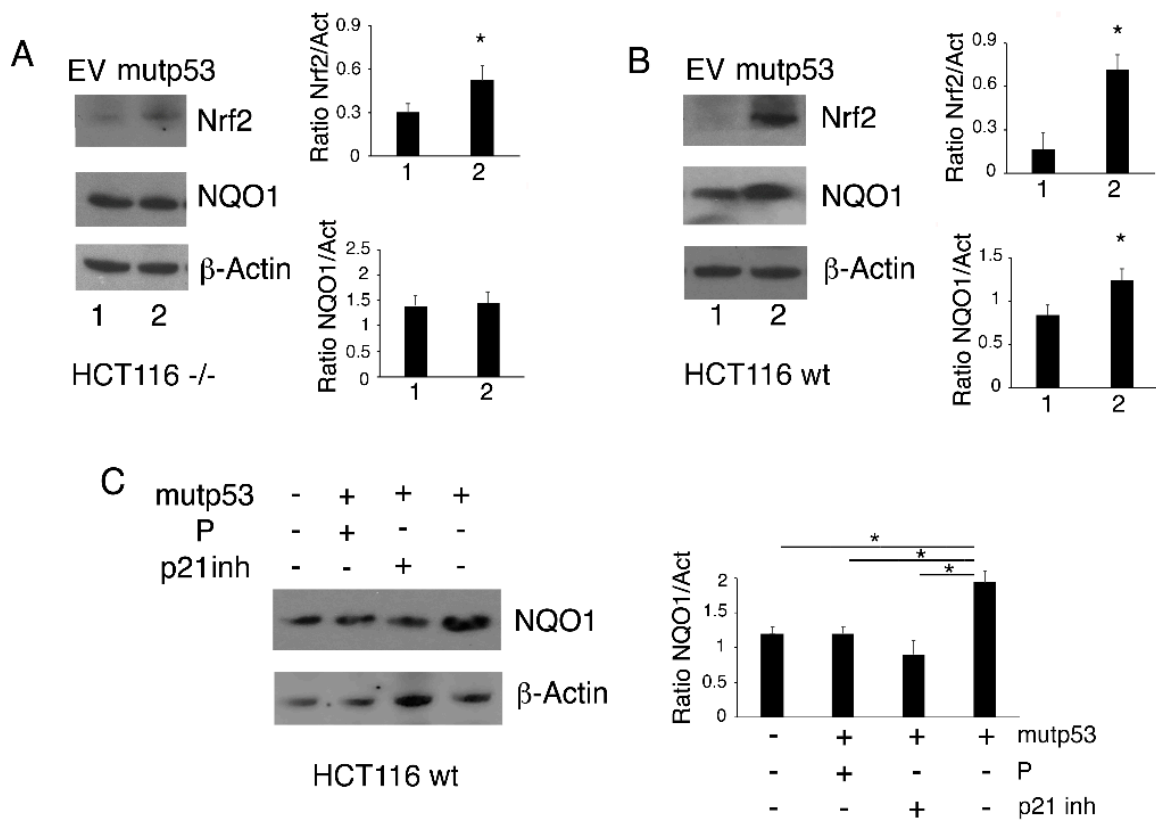

Figure 6. The Nrf2/NQO1 axis is activated in HCT116 p53-/- cells but not in HCT116 wtp53 cells. HCT116 p53-/- and HCT116 wtp53 cells were transfected with empty vector (EV, 1) or the pcDNA3p53R273H vector (mutp53, 2). (A) and (B) After 24 h, Nrf2 and NQO1 expression were evaluated by Western blot analysis. Actin was used as the loading control. The histograms represent the mean + SD of the densitometric analysis of the ratio between Nrf2 or NQO1 and actin. * $p$ value $<0.05$. (C) NQO1 expression was evaluated by Western blot in HCT116 wtp53 cells pre-treated with pifithrin- $\alpha(\mathrm{P})$ $(30 \mu \mathrm{M})$ or UC2288-p21 inhibitor (p21 inh) $(5 \mu \mathrm{M})$ and then transfected as described above. Control cells were untreated. The histograms represent the mean $+\mathrm{SD}$ of the densitometric analysis of the ratio between NQO1 and actin. ${ }^{*} p$ value $<0.05$.

Finally, to evaluate the role of the wtp53/21 axis in NQO1 upregulation, we used the p53 inhibitor pifithrin- $\alpha$ or the p21 inhibitor UC2288 in HCT116 wtp53 cells following $p 53-R 273 H$ transfection and found that both treatments counteracted the increase in NQO1 expression induced by $p 53-\mathrm{R} 273 \mathrm{H}$ transfection (Figure 6C).

Altogether, these results suggest that the antioxidant response activated by the wtp53/p21 axis prevented the ROS increase induced by p53-R273H expression in cells carrying wtp53.

\section{Discussion}

In this study, we found that p53-R273H promoted cell survival by increasing ROS and promoting cytokine release in cancer cells lacking wtp53, and these effects were counter- 
acted by the presence of wtp53. This could explain why cancers carrying homozygous mutp53 display more aggressive behaviors and why cancer cells tend to lose the wtp53 allele during malignant progression [30]. We found that the differences in intracellular ROS level observed in p53-/- HCT116 cells and wtp53-carrying HCT116 cells following p53-R273H transfection correlated with the downregulation of UCP2 expression level in cancer cells lacking wtp53. UCP2 is a protein that can help to dissipate the proton gradient and prevent the proton-motive force from becoming excessive, reducing the ROS produced by electron transport [31]. Interestingly, UCP2 has been also reported to help to preserve mitochondrial integrity by preventing the loss of membrane potential [32], and thus its downregulation by $\mathrm{p} 53-\mathrm{R} 273 \mathrm{H}$ could increase mitochondrial damage and $\mathrm{ROS}$ production.

The downregulation of the AMPK/PGC- $1 \alpha / \mathrm{UCP} 2$ axis has previously been shown to strongly contribute to mutp53 ROS increase and the subsequently induced oncogenesis [26]. Interestingly, here we found that UCP2 was downregulated following p53R273H-transfection only in cancer cells lacking wtp53 in which mTOR activation was also observed.

Another important finding of this study was that to further increase ROS, p53-R273H transfection reduced both autophagy and mitophagy in these cells. The dysregulation of these processes plays an important role in cancer, although autophagy may play different roles depending on the stage of carcinogenesis [33]. The reduction of mitophagy by mutp53 transfection in cells lacking wtp53 represents a new mechanism through which p53-R273H could promote ROS production and sustain cell survival.

The antioxidant response, mainly regulated by the transcription factor Nrf2, plays a key role in the control of ROS. We found that it was upregulated following p53-R273H transfection independently of p53 status. However, according to a study showing that mutp53 can tune Nrf2 transcription activity, resulting in the upregulation of specific targets [28], here we found that NQO1, antioxidant molecular target of Nrf2, was upregulated only in cancer cells carrying wtp53.

In cells lacking wtp53, Nrf2 upregulation could be due to the positive feedback loop induced with mutp53 [34], while in the case of cancer cells carrying wtp53, p53-R273H transfection could lead to the upregulation of Nrf2 through the activation of the wtp53/p21 axis. Indeed, in response to oxidative stress, p21 may interact with Nrf2 and compete with Kelch-like ECH-associated protein 1 (Keap1) for binding to this transcription factor, preventing its degradation and thus increasing its stabilization [35]. In agreement with these findings, here we observed that p53-R273H transfection upregulated p21 in wtp53-carrying cells, and that its inhibition by UC2288 as well as the inhibition of wtp53 by pifithrin- $\alpha$ prevented NQO1 upregulation. Although cancer cells also upregulate the antioxidant response to avoid increases so high that they could cause cell death, ROS increases may promote tumorigenesis by several means, particularly due to their interplay with the inflammatory cytokines and oncogenic pathways that regulate their release [36,37]. These cytokines may, in turn, increase ROS production in a positive regulatory circuit that amplifies pro-tumorigenic inflammation $[37,38]$. Oxidative stress may promote carcinogenesis by several other mechanisms, i.e., by favoring DNA damage and negatively modulating the DNA damage repair response (DDR) [39]. The DDR has been shown to be reduced by mutp53 through the impairment of ataxia-telangiectasia mutated (ATM) activation [40]; therefore, it will be interesting to investigate if its mediated increase in ROS could contribute to such an effect. According to the findings that conversely to mutp53, wtp53 may inhibit the activation of several pro-survival pathways and counteract inflammatory cytokine release, in this study, we found that in wtp53-carrying cancer cells, the activation of p21 following p53-R273H transfection counteracted cell proliferation, ROS production and cytokine release. This suggests that despite the dominant negative effect exerted by mutp53 on wtp53, the activation of the latter may still restrain all pro-tumorigenic effects that we found in this study to be induced by p53-R273H.

In conclusion, this study highlights that ROS increase (due to several mechanisms including the dysregulation of bulk autophagy, mitophagy and UCP2 downregulation) plays a key role 
in the pro-survival effect induced by $\mathrm{p} 53-\mathrm{R} 273 \mathrm{H}$, in correlation with $m$-TOR activation and pro-inflammatory/immune suppressive cytokine release. This study also unveils the capacity of wtp53 to restrain these pro-tumorigenic effects induced by p53- $2273 \mathrm{H}$ transfection.

Author Contributions: M.A.R.: investigation, visualization, methodology, formal analysis. M.S.G.M.: data curation, formal analysis, investigation, validation, writing-review and editing. R.B.: software, investigation, formal analysis. A.A. visualization, formal analysis, methodology. G.D.: conceptualization, writing-review and editing. M.C.: conceptualization, resources, data curation, formal analysis, funding acquisition, validation, project administration, writing-review and editing. All authors have read and agreed to the published version of the manuscript.

Funding: This work was supported by grants from the Istituto Pasteur Italia-Fondazione Cenci Bolognetti, PRIN 2017 (2017K55HLC) and by the Italian Association for Cancer Research (AIRC; grant IG 2019 Id.23040).

Institutional Review Board Statement: Not applicable.

Informed Consent Statement: Not applicable.

Data Availability Statement: The datasets generated and/or analyzed during the current study are available from the corresponding author upon reasonable request.

Conflicts of Interest: The authors declare no conflict of interest.

\section{References}

1. D'Orazi, G.; Cirone, M. Mutant p53 and Cellular Stress Pathways: A Criminal Alliance That Promotes Cancer Progression. Cancers 2019, 11, 614. [CrossRef]

2. Dando, I.; Cordani, M.; Donadelli, M. Mutant p53 and mTOR/PKM2 regulation in cancer cells. IUBMB Life 2016, 68, 722-726. [CrossRef]

3. Romeo, M.A.; Gilardini Montani, M.S.; Benedetti, R.; Santarelli, R.; D'Orazi, G.; Cirone, M. STAT3 and mutp53 Engage a Positive Feedback Loop Involving HSP90 and the Mevalonate Pathway. Front. Oncol. 2020, 10, 1102. [CrossRef] [PubMed]

4. Parrales, A.; Thoenen, E.; Iwakuma, T. The interplay between mutant p53 and the mevalonate pathway. Cell Death Differ. 2018, 25, 460-470. [CrossRef]

5. Ingallina, E.; Sorrentino, G.; Bertolio, R.; Lisek, K.; Zannini, A.; Azzolin, L.; Severino, L.U.; Scaini, D.; Mano, M.; Mantovani, F.; et al. Mechanical cues control mutant p53 stability through a mevalonate-RhoA axis. Nat. Cell Biol. 2018, 20, 28-35. [CrossRef] [PubMed]

6. Cordani, M.; Butera, G.; Pacchiana, R.; Masetto, F.; Mullappilly, N.; Riganti, C.; Donadelli, M. Mutant p53-Associated Molecular Mechanisms of ROS Regulation in Cancer Cells. Biomolecules 2020, 10, 361. [CrossRef]

7. Ma, L.; Fu, Q.; Xu, B.; Zhou, H.; Gao, J.; Shao, X.; Xiong, J.; Gu, Q.; Wen, S.; Li, F.; et al. Breast cancer-associated mitochondrial DNA haplogroup promotes neoplastic growth via ROS-mediated AKT activation. Int. J. Cancer 2018, 142, 1786-1796. [CrossRef] [PubMed]

8. Granato, M.; Rizzello, C.; Gilardini Montani, M.S.; Cuomo, L.; Vitillo, M.; Santarelli, R.; Gonnella, R.; D'Orazi, G.; Faggioni, A.; Cirone, M. Quercetin induces apoptosis and autophagy in primary effusion lymphoma cells by inhibiting PI3K/AKT/mTOR and STAT3 signaling pathways. J. Nutr. Biochem. 2017, 41, 124-136. [CrossRef]

9. Bhatt, A.P.; Bhende, P.M.; Sin, S.H.; Roy, D.; Dittmer, D.P.; Damania, B. Dual inhibition of PI3K and mTOR inhibits autocrine and paracrine proliferative loops in PI3K/Akt/mTOR-addicted lymphomas. Blood 2010, 115, 4455-4463. [CrossRef] [PubMed]

10. D'Orazi, G.; Cordani, M.; Cirone, M. Oncogenic pathways activated by pro-inflammatory cytokines promote mutant p53 stability: Clue for novel anticancer therapies. Cell. Mol. Life Sci. 2020. [CrossRef]

11. Moon, S.H.; Huang, C.H.; Houlihan, S.L.; Regunath, K.; Freed-Pastor, W.A.; Morris, J.P.t.; Tschaharganeh, D.F.; Kastenhuber, E.R.; Barsotti, A.M.; Culp-Hill, R.; et al. p53 Represses the Mevalonate Pathway to Mediate Tumor Suppression. Cell 2019, 176, 564-580.e519. [CrossRef] [PubMed]

12. Sorrentino, G.; Mantovani, F.; Del Sal, G. The stiff RhoAd from mevalonate to mutant p53. Cell Death Differ. 2018, 25, 645-647. [CrossRef] [PubMed]

13. Granato, M.; Gilardini Montani, M.S.; Santarelli, R.; D'Orazi, G.; Faggioni, A.; Cirone, M. Apigenin, by activating p53 and inhibiting STAT3, modulates the balance between pro-apoptotic and pro-survival pathways to induce PEL cell death. J. Exp. Clin. Cancer Res. 2017, 36, 167. [CrossRef]

14. Feng, Z. p53 regulation of the IGF-1/AKT/mTOR pathways and the endosomal compartment. Cold Spring Harb. Perspect. Biol. 2010, 2, a001057. [CrossRef] [PubMed]

15. He, Z.; Simon, H.U. A novel link between p53 and ROS. Cell Cycle 2013, 12, 201-202. [CrossRef] 
16. Boudreau, H.E.; Ma, W.F.; Korzeniowska, A.; Park, J.J.; Bhagwat, M.A.; Leto, T.L. Histone modifications affect differential regulation of TGFbeta- induced NADPH oxidase 4 (NOX4) by wild-type and mutant p53. Oncotarget 2017, 8, 44379-44397. [CrossRef]

17. Desaint, S.; Luriau, S.; Aude, J.C.; Rousselet, G.; Toledano, M.B. Mammalian antioxidant defenses are not inducible by H2O2. J. Biol. Chem. 2004, 279, 31157-31163. [CrossRef] [PubMed]

18. Rotblat, B.; Melino, G.; Knight, R.A. NRF2 and p53: Januses in cancer? Oncotarget 2012, 3, 1272-1283. [CrossRef]

19. D'Orazi, G.; Garufi, A.; Cirone, M. Nuclear factor erythroid 2 (NF-E2) p45-related factor 2 interferes with homeodomain-interacting protein kinase 2/p53 activity to impair solid tumors chemosensitivity. IUBMB Life 2020, 72, 1634-1639. [CrossRef]

20. Tasdemir, E.; Chiara Maiuri, M.; Morselli, E.; Criollo, A.; D’Amelio, M.; Djavaheri-Mergny, M.; Cecconi, F.; Tavernarakis, N.; Kroemer, G. A dual role of p53 in the control of autophagy. Autophagy 2008, 4, 810-814. [CrossRef]

21. Cecchinelli, B.; Lavra, L.; Rinaldo, C.; Iacovelli, S.; Gurtner, A.; Gasbarri, A.; Ulivieri, A.; Del Prete, F.; Trovato, M.; Piaggio, G.; et al. Repression of the antiapoptotic molecule galectin-3 by homeodomain-interacting protein kinase 2-activated p53 is required for p53-induced apoptosis. Mol. Cell. Biol. 2006, 26, 4746-4757. [CrossRef]

22. Cordani, M.; Oppici, E.; Dando, I.; Butturini, E.; Dalla Pozza, E.; Nadal-Serrano, M.; Oliver, J.; Roca, P.; Mariotto, S.; Cellini, B.; et al. Mutant p53 proteins counteract autophagic mechanism sensitizing cancer cells to mTOR inhibition. Mol. Oncol. 2016, 10, 1008-1029. [CrossRef] [PubMed]

23. Pitolli, C.; Wang, Y.; Mancini, M.; Shi, Y.; Melino, G.; Amelio, I. Do Mutations Turn p53 into an Oncogene? Int. J. Mol. Sci. 2019, 20, 6241. [CrossRef]

24. Rocha, S.; Campbell, K.J.; Perkins, N.D. p53- and Mdm2-independent repression of NF-kappa B transactivation by the ARF tumor suppressor. Mol. Cell 2003, 12, 15-25. [CrossRef]

25. Santarelli, R.; Arteni, A.M.B.; Gilardini Montani, M.S.; Romeo, M.A.; Gaeta, A.; Gonnella, R.; Faggioni, A.; Cirone, M. KSHV dysregulates bulk macroautophagy, mitophagy and UPR to promote endothelial to mesenchymal transition and CCL2 release, key events in viral-driven sarcomagenesis. Int. J. Cancer 2020, 147, 3500-3510. [CrossRef] [PubMed]

26. Cordani, M.; Butera, G.; Dando, I.; Torrens-Mas, M.; Butturini, E.; Pacchiana, R.; Oppici, E.; Cavallini, C.; Gasperini, S.; Tamassia, N.; et al. Mutant p53 blocks SESN1/AMPK/PGC-1alpha/UCP2 axis increasing mitochondrial O2-. production in cancer cells. Br. J. Cancer 2018, 119, 994-1008. [CrossRef]

27. Dando, I.; Pacchiana, R.; Pozza, E.D.; Cataldo, I.; Bruno, S.; Conti, P.; Cordani, M.; Grimaldi, A.; Butera, G.; Caraglia, M.; et al. UCP2 inhibition induces ROS/Akt/mTOR axis: Role of GAPDH nuclear translocation in genipin/everolimus anticancer synergism. Free Radic. Biol. Med. 2017, 113, 176-189. [CrossRef]

28. Lisek, K.; Campaner, E.; Ciani, Y.; Walerych, D.; Del Sal, G. Mutant p53 tunes the NRF2-dependent antioxidant response to support survival of cancer cells. Oncotarget 2018, 9, 20508-20523. [CrossRef]

29. Kalo, E.; Kogan-Sakin, I.; Solomon, H.; Bar-Nathan, E.; Shay, M.; Shetzer, Y.; Dekel, E.; Goldfinger, N.; Buganim, Y.; Stambolsky, P.; et al. Mutant p53R273H attenuates the expression of phase 2 detoxifying enzymes and promotes the survival of cells with high levels of reactive oxygen species. J. Cell Sci. 2012, 125, 5578-5586. [CrossRef]

30. Williams, A.B.; Schumacher, B. p53 in the DNA-Damage-Repair Process. Cold Spring Harb. Perspect. Med. 2016, 6. [CrossRef]

31. Garlid, K.D.; Jaburek, M.; Jezek, P.; Varecha, M. How do uncoupling proteins uncouple? Biochim. Biophys. Acta 2000, 1459, 383-389. [CrossRef]

32. Qin, N.; Cai, T.; Ke, Q.; Yuan, Q.; Luo, J.; Mao, X.; Jiang, L.; Cao, H.; Wen, P.; Zen, K.; et al. UCP2-dependent improvement of mitochondrial dynamics protects against acute kidney injury. J. Pathol. 2019, 247, 392-405. [CrossRef]

33. Cirone, M.; Gilardini Montani, M.S.; Granato, M.; Garufi, A.; Faggioni, A.; D'Orazi, G. Autophagy manipulation as a strategy for efficient anticancer therapies: Possible consequences. J. Exp. Clin. Cancer Res. 2019, 38, 262. [CrossRef]

34. Gilardini Montani, M.S.; Cecere, N.; Granato, M.; Romeo, M.A.; Falcinelli, L.; Ciciarelli, U.; D’Orazi, G.; Faggioni, A.; Cirone, M. Mutant p53, Stabilized by Its Interplay with HSP90, Activates a Positive Feed-Back Loop Between NRF2 and p62 that Induces Chemo-Resistance to Apigenin in Pancreatic Cancer Cells. Cancers 2019, 11, 703. [CrossRef] [PubMed]

35. Chen, W.; Sun, Z.; Wang, X.J.; Jiang, T.; Huang, Z.; Fang, D.; Zhang, D.D. Direct interaction between Nrf2 and p21(Cip1/WAF1) upregulates the Nrf2-mediated antioxidant response. Mol. Cell 2009, 34, 663-673. [CrossRef] [PubMed]

36. Kumari, S.; Badana, A.K.; Malla, R. Reactive Oxygen Species: A Key Constituent in Cancer Survival. Biomark. Insights 2018, 13, 1177271918755391. [CrossRef]

37. Naik, E.; O’Reilly, L.A.; Asselin-Labat, M.L.; Merino, D.; Lin, A.; Cook, M.; Coultas, L.; Bouillet, P.; Adams, J.M.; Strasser, A. Destruction of tumor vasculature and abated tumor growth upon VEGF blockade is driven by proapoptotic protein Bim in endothelial cells. J. Exp. Med. 2011, 208, 1351-1358. [CrossRef]

38. Yang, D.; Elner, S.G.; Bian, Z.M.; Till, G.O.; Petty, H.R.; Elner, V.M. Pro-inflammatory cytokines increase reactive oxygen species through mitochondria and NADPH oxidase in cultured RPE cells. Exp. Eye Res. 2007, 85, 462-472. [CrossRef]

39. Gruosso, T.; Mieulet, V.; Cardon, M.; Bourachot, B.; Kieffer, Y.; Devun, F.; Dubois, T.; Dutreix, M.; Vincent-Salomon, A.; Miller, K.M.; et al. Chronic oxidative stress promotes $\mathrm{H} 2 \mathrm{AX}$ protein degradation and enhances chemosensitivity in breast cancer patients. EMBO Mol. Med. 2016, 8, 527-549. [CrossRef] [PubMed]

40. Song, H.; Hollstein, M.; Xu, Y. p53 gain-of-function cancer mutants induce genetic instability by inactivating ATM. Nat. Cell Biol. 2007, 9, 573-580. [CrossRef] 